\section{Estimation of the Anterior Extent of the Meyer Loop Using MR Tractography}

We read with interest the recent study by Yamamoto et al in the American Journal of Neuroradiology. ${ }^{1}$ The authors aimed to determine the optimal directional motion-probing gradient (MPG) setting for imaging the optic radiations by means of MR tractography by evaluating images captured with multiple settings. Although the authors found no advantage to altering MPG settings, they successfully visualized the optic radiations in 20 of the 24 hemispheres examined. Their findings add support to existing studies and further demonstrate the efficacy of MR tractography in imaging the trajectory of the optic radiations. We congratulate the team on their careful study and useful addition to the literature but wondered whether they were able to extrapolate measurements from their images, which might aid our understanding of the anatomic variations in Meyer loop that exist between patients.

Anterior temporal lobe (ATL) resections are increasingly being performed for medically refractory temporal lobe epilepsy but carry a significant risk of contralateral superior quadrant visual field defects due to disruption of the anterior fibers of the Meyer loop. The extent of postoperative field defects can vary significantly between patients, despite comparable resection sizes, and is most likely due to intersubject variability in the anterior limit of the Meyer loop. On recent examination of our series of patients with epilepsy undergoing ATL resections, $25 \%$ of patients undergoing a standard $3-$ to $4.5-\mathrm{cm}$ en bloc resection developed a significant upper quadrantanopia, $8 \%$ developed a homonomous hemianopia, and $67 \%$ were unaffected. Improving our understanding of the range of anatomic variance, which might explain these differences in outcomes, would, therefore, be of use.

To date, much of our understanding of the anatomy of the Meyer loop has come from studies correlating the extent and pattern of field defects with resection lengths following temporal lobectomy. Most studies have relied on qualitative or semiquantitative data for their analysis. The first quantitative estimation of the anterior limit of the Meyer loop was made by Barton et al in 2005, ${ }^{2}$ whose estimations were based on a linear regression model, which assumes that the linear relationship between field defects and resection lengths is continuous. This may not be the case.

MR tractography is a promising technique and is likely to have applications in the future in preoperative planning for temporal lobe surgery. Although some studies have attempted to demonstrate its use in predicting field defects, ${ }^{3,4}$ it has yet to be validated on any meaningful scale in clinical practice for this purpose. In the meantime, future investigations using MR tractography to make measurements of the anterior limits of the Meyer loop from the temporal pole and to describe the anatomic variance between patients would be welcomed and would provide a useful and informative update to the existing literature.

\section{References}

1. Yamamoto A, Miki Y, Urayama S, et al. Diffusion tensor fiber tractography of the optic radiation: analysis with 6-, 12-, 40-, and 81-directional motion-probing gradients-a preliminary study. AJNR Am J Neuroradiol 2007;28:92-96

2. Barton JJ, Hefter R, Chang B, et al. The field defects of anterior temporal lobectomy: a quantitative reassessment of Meyer's loop. Brain 2005;128:212333. Epub 2005 May 25

3. Taoka T, Sakamoto M, Iwasaki S, et al. Diffusion tensor imaging in cases with visual field defect after anterior temporal lobectomy. AJNR Am J Neuroradiol 2005;26:797-803

4. Powell HWR, Parker GJM, Alexander DC, et al. MR tractography predicts visual field defects following temporal lobe resection. Neurology 2005;65:596-99

$$
\begin{array}{r}
\text { K. Abdel-Aziz } \\
\text { P. Goulding } \\
\text { Department of Neurology } \\
\text { Leeds General Infirmary } \\
\text { Leeds, UK }
\end{array}
$$

DOI 10.3174/ajnr.A0889 